\title{
SUCRALOSE NO DESENVOLVIMENTO DE SOBREMESAS LÁCTEAS LIGHT
}

\author{
ELLEN PORTO PINTO * \\ ANDRÉA MIRANDA TEIXEIRA * \\ LETÍCIA LOPES SOPEÑA * \\ VANESSA PIRES DA ROSA * \\ MÁRCIA DE MELLO LUVIELMO **
}

\begin{abstract}
Avaliou-se o efeito do edulcorante sucralose em sobremesa láctea tipo mousse, verificando a redução calórica e a aceitabilidade do produto obtido. Pesquisa de mercado evidenciou preferência por sobremesa láctea nos sabores chocolate ou maracujá, que fosse consistente, muito aerada, nas versões light ou convencional. Foram desenvolvidas quatro formulações, nos dois sabores preferidos (chocolate e maracujá), nas versões convencional e light. As formulações que obtiveram maior índice de aceitação foram chocolate convencional e chocolate light. Como resultados foram obtidos índices de aceitação de $89,9 \%$ e $79,2 \%$ e teores calóricos de 226,09 kcal/kg e 154,10 kcal/kg para o mousse de chocolate convencional e light, respectivamente. Concluiu-se pela viabilidade da elaboração de sobremesa pronta tipo mousse light utilizando sucralose (redução calórica de $31,8 \%)$.
\end{abstract}

PALAVRAS-CHAVE: MOUSSE; SUCRALOSE; SOBREMESA LÁCTEA LIGHT.

\section{INTRODUÇÃO}

A ampliação da expectativa média de vida das pessoas deve-se aos avanços da medicina, ao desenvolvimento de hábitos mais saudáveis e à prevenção de doenças como hipertensão, arteriosclerose, diabetes e câncer.

* Químico de Alimentos, Departamento de Ciência dos Alimentos, Universidade Federal de Pelotas (UFPEL), RS.

** Engenheira de Alimentos, Mestre em Nutrição Aplicada a Tecnologia de Alimentos, Faculdade de Engenharia de Alimentos, Universidade Estadual de Campinas (UNICAMP), SP. (e-mail: luvielmo@fea.unicamp.br). 
A indústria de alimentos, atenta ao mercado consumidor, tem desenvolvido produtos e tecnologias para a produção de alimentos com baixas calorias, mediante substituição de açúcar por edulcorantes e/ou redução no seu teor de gordura (CÂNNDIDO e CAMPOS, 1996).

De acordo com a Portaria 27/98, da Secretaria de Vigilância Sanitária do Ministério da Saúde, produtos light são aqueles que apresentam redução de pelo menos $25 \%$ do valor calórico pela substituição ou redução de alguns de seus componentes (BRASIL, 1998). Na prática, a energia que os alimentos fornecem (valor energético total) é estimada pela determinação do teor de proteínas, lipídios e carboidratos, utilizando-se para os cálculos os valores de $4 \mathrm{kcal} / \mathrm{g}$ para proteínas e carboidratos, e $9 \mathrm{kcal} / \mathrm{g}$ para gorduras. A soma dos valores individuais dos três macronutrientes fornece o valor calórico aproximado do alimento. Assim, a redução ou substituição de um desses nutrientes permite diminuir 0 valor energético total nos alimentos (CÂNDIDO e CAMPOS, 1996).

Os produtos light normalmente são elaborados substituindo-se o açúcar por edulcorantes, havendo ainda grande demanda por alimentos com substituição parcial ou total da gordura por substâncias que conferem consistência como carboximetilcelulose, pectinas, polidextrinas e outros (VARNAM e SUTHERLAND, 1995).

A sucralose, edulcorante não-calórico de última geração, é obtido a partir da sacarose. Apresenta características singulares, entre as quais alto poder edulcorante (600 vezes mais doce que a sacarose) e alta estabilidade (CAMPOS, 2002). Sua doçura, de percepção rápida, persiste por período ligeiramente maior que o da sacarose sem revelar residual amargo ou metálico. Apresenta alta solubilidade em água e estabilidade térmica em meio aquoso ou ácido, bem como durante armazenamento. É compatível com outros ingredientes alimentícios, incluindo flavorizantes, temperos e conservantes (CÂNDIDO e CAMPOS, 1996).

Estudos têm demonstrado de maneira definitiva que a sucralose é inócua, mesmo em níveis de consumo muito superiores ao normal, não havendo nenhum tipo de restrição ao seu consumo (CAMPOS, 2000). Pode ser ingerida por diabéticos, pois não há indicação de que estimule a secreção da insulina ou reduza a concentração de glicose no plasma. Também não gera efeitos teratogênicos ou mutagênicos (CÂNDIDO e CAMPOS, 1996).

O presente estudo teve por objetivo avaliar o efeito do edulcorante sucralose em sobremesa láctea tipo mousse, verificando-se o grau de redução calórica e a aceitabilidade do produto desenvolvido. 


\section{MATERIAL E MÉTODOS}

\subsection{MATERIAL}

As matérias-primas utilizadas para elaboração dos mousses foram: chocolate ao leite em barra (Nestlé Brasil Ltda.), creme de leite (Nestlé Brasil Ltda.), cacau em pó (Nestlé Brasil Ltda.), gelatina em pó (Produtos Alimentícios Fleischmann e Royal Ltda.), carboximetilcelulose (Arco-Íris Brasil Ind. e Com. de Produtos Alimentícios Ltda), edulcorante Sucralose Splenda (Johnson \& Johnson), leite condensado (Nestlé Brasil Ltda.), suco concentrado sabor maracujá (Fleischmann Royal Nabisco), clara de ovos (Companhia Minuano de Alimentos) e leite desnatado (Cooperativa Sul-Rio Grandense de Laticínios Ltda.).

\subsection{MÉTODOS}

\subsubsection{Pesquisa de Mercado}

Realizou-se pesquisa de mercado com a população do Campus Universitário (Universidade Federal de Pelotas - localizado em Capão do Leão/RS), mediante questionário com perguntas objetivas e abordagem direta, a fim de determinar as características das sobremesas a serem testadas. Utilizou-se amostragem simples e inspeção comum, com nível geral de inspeção dois, que corresponde à amostra de 200 pessoas conforme a Norma ABC - STD - 105D (LOURENÇO FILHO, 1984).

\subsubsection{Elaboração do Produto}

A elaboração das sobremesas lácteas, nas versões convencional e light, foi efetuada em pequena escala conforme fluxograma de fabricação (Figuras 1 a 4$)$.

\subsubsection{Análise Sensorial}

$\mathrm{Na}$ avaliação sensorial das sobremesas lácteas foi utilizado teste de aceitação, com escala hedônica de nove pontos e 105 julgadores não treinados, conforme metodologia de TEIXEIRA, MEINERT e BARBITTA (1987). Os cálculos de análise de variância e teste de Tukey para comparação de médias foram realizados utilizando-se o software Statistica 5.0 (STATSOFT, 1999). 
FIGURA 1 - FLUXOGRAMA DE ELABORAÇÃO DE MOUSSE CONVENCIONAL DE CHOCOLATE

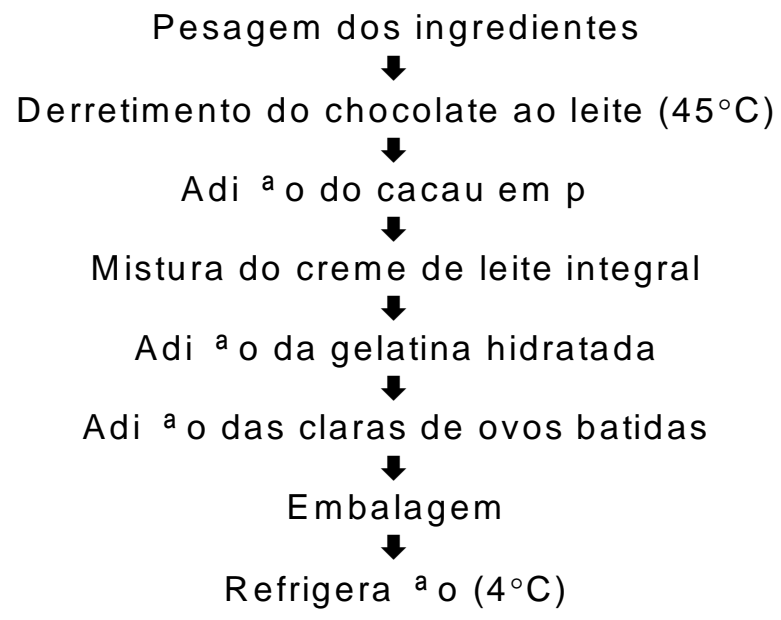

\section{FIGURA 2 - FLUXOGRAMA DE ELABORAÇÃO DE MOUSSE LIGHT DE CHOCOLATE}

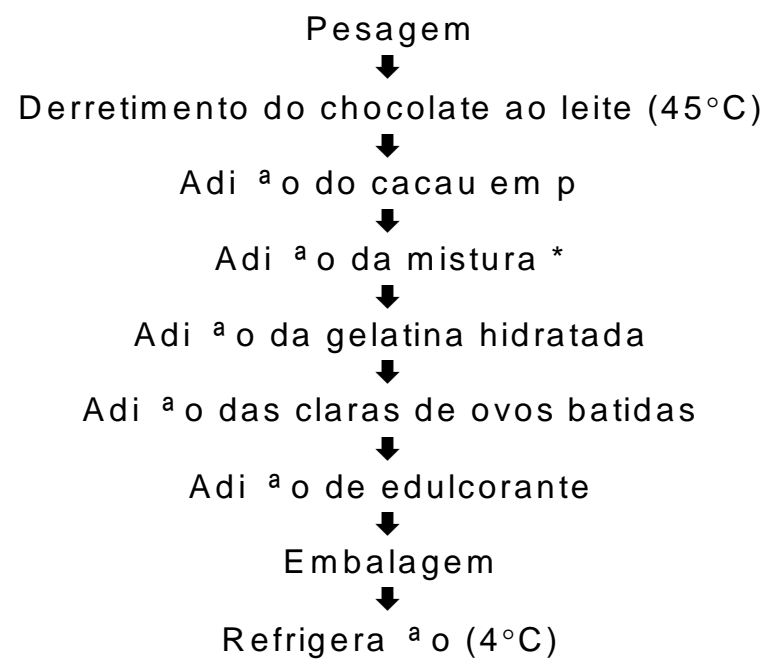

* Mistura: Carboximetilcelulose dissolvido, leite desnatado e creme de leite integral. 
FIGURA 3 - FLUXOGRAMA DE ELABORAÇÃO DE MOUSSE CONVENCIONAL DE MARACUJÁ

\title{
Pesagem dos ingredientes
}

Mistura do leite condensado, creme de leite integral e suco concentrado

\author{
Adi $\stackrel{a}{\circ}$ da gelatina hidratada \\ Mistura das claras de ovos batidas \\ Embalagem \\ Refrigera ${ }^{a} \circ\left(4^{\circ} \mathrm{C}\right)$
}

\section{FIGURA 4 - FLUXOGRAMA DE ELABORAÇÃO DE MOUSSE LIGHT DE MARACUJÁ}

\author{
Mistura do leite condensado e suco \\ concentrado de maracujÆ \\ $\checkmark$ \\ Adi $\stackrel{a}{\circ}$ da mistura * \\ Adi a o da gelatina hidratada \\ $\checkmark$ \\ Mistura das claras de ovos batidas \\ Embalagem \\ $\checkmark$ \\ Refrigera ${ }^{a} \circ\left(4^{\circ} \mathrm{C}\right)$
}

* Mistura: Carboximetilcelulose dissolvido, leite desnatado e creme de leite. 


\subsubsection{Composição Centesimal}

Determinou-se a composição centesimal das duas formulações com maior índice de aceitabilidade de acordo com a AOAC (1987). A partir dos resultados calculou-se o valor calórico total de cada produto, segundo metodologia de OSBORNE e VOOGT (1978).

\section{RESULTADOS E DISCUSSÃO}

\subsection{RESULTADO DA PESQUISA DE MERCADO}

A pesquisa de mercado revelou que o consumidor prefere sobremesa láctea nos sabores chocolate ou maracujá (Figura 5), que seja consistente (Figura 6) e muito aerada (Figura 7), nas versões light ou convencional (Figura 8).

\section{FIGURA 5- PREFERÊNCIA DOS CONSUMIDORES POR SOBREMESAS LÁCTEAS DE ACORDO COM O ATRIBUTO SABOR}

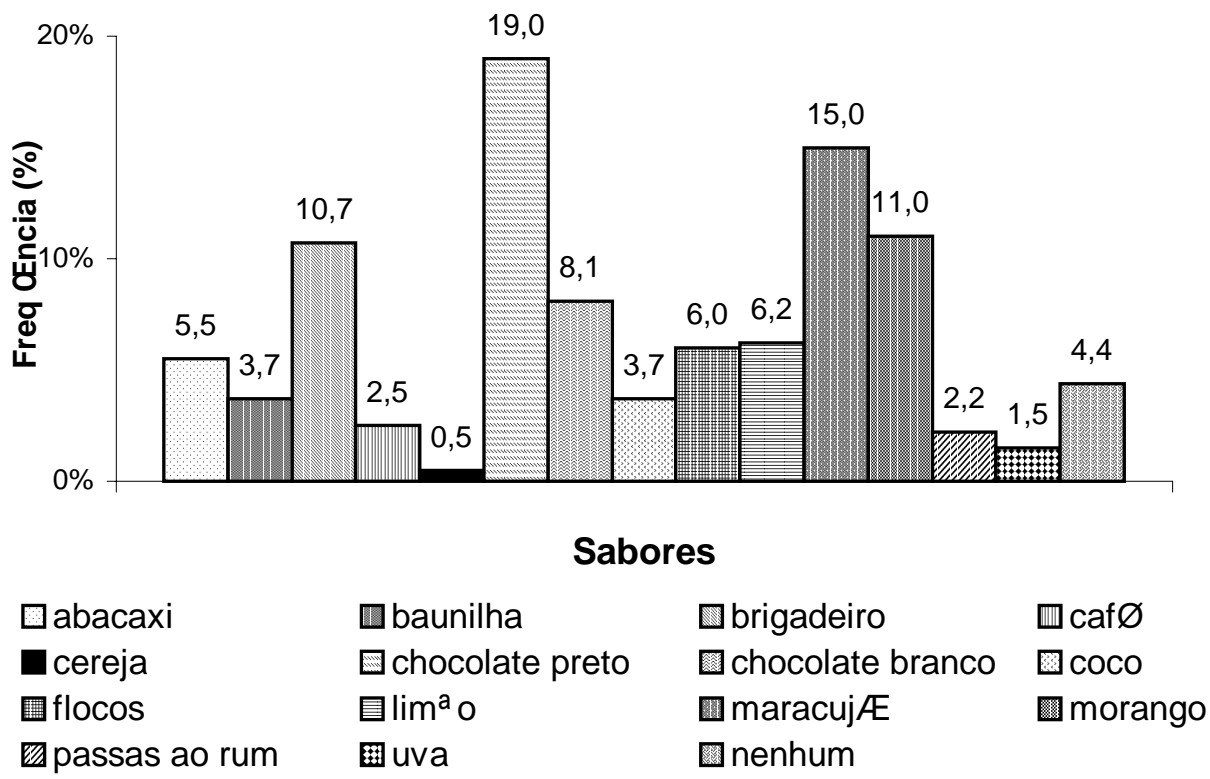


FIGURA 6- PREFERÊNCIA DOS CONSUMIDORES POR SOBREMESAS LÁCTEAS SEGUNDO A CONSISTÊNCIA DO PRODUTO

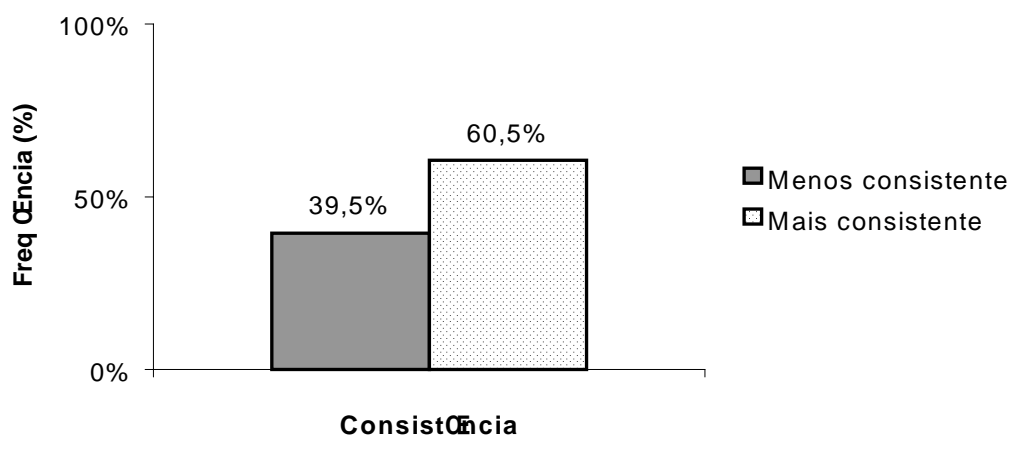

FIGURA 7-PREFERÊNCIA DOS CONSUMIDORES POR SOBREMESAS LÁCTEAS SEGUNDO A TEXTURA DO PRODUTO

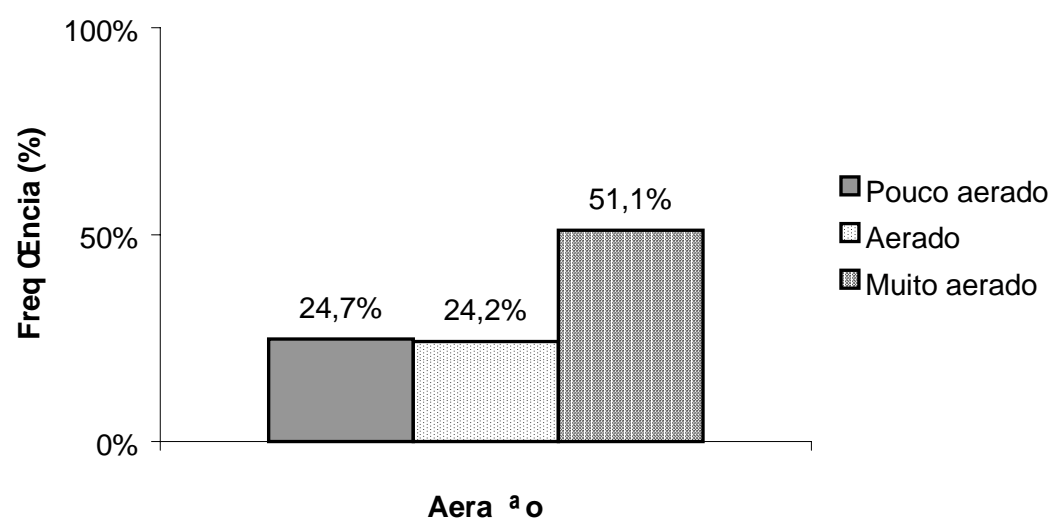




\section{FIGURA 8 - PREFERÊNCIA DOS CONSUMIDORES POR SOBREMESAS LÁCTEAS NAS VERSÕES LIGHT OU CONVENCIONAL}

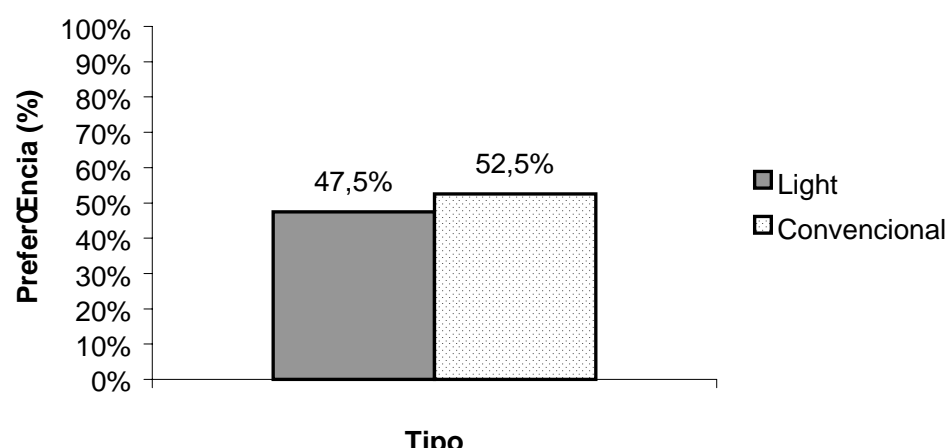

Visando atender as características levantadas na pesquisa de mercado foram elaboradas duas sobremesas lácteas, nos sabores chocolate e maracujá, nas versões light e convencional.

\subsection{RESULTADO DA ANÁLISE SENSORIAL}

Os resultados do teste de aceitação das sobremesas de chocolate e maracujá, nas versões light e convencional, constam da Tabela 1.

\section{TABELA 1 - MÉDIAS E DESVIOS OBTIDOS NO TESTE DE ACEITAÇÃO DAS SOBREMESAS LÁCTEAS}

\begin{tabular}{lcc}
\hline Amostras & MØdias & $\begin{array}{c}\text { Desvio } \\
\text { Padr } \mathrm{o}\end{array}$ \\
\hline Chocolate & $8,09 \mathrm{a}$ & 1,04 \\
convencional & $7,13 \mathrm{~b}$ & 1,43 \\
Chocolate light & $7,29 \mathrm{~b}$ & 1,73 \\
Maracuj/E & $7,14 \mathrm{~b}$ & 1,62 \\
convencional & & \\
Maracuj/ヨight & & \\
\hline
\end{tabular}


O teste de Tukey, ao nível de $0,05 \%$ de significância $(p=0,000002)$, evidenciou que o mousse de chocolate convencional diferiu significativamente das demais amostras, as quais não diferiram entre si. Escolheu-se o mousse de chocolate convencional para continuação do trabalho (Figura 9) em razão de ter alcançado o maior índice de aceitabilidade $(89,95 \%)$.

\section{FIGURA 9 - ÍNDICE DE ACEITABILIDADE DAS SOBREMESAS LÁCTEAS}

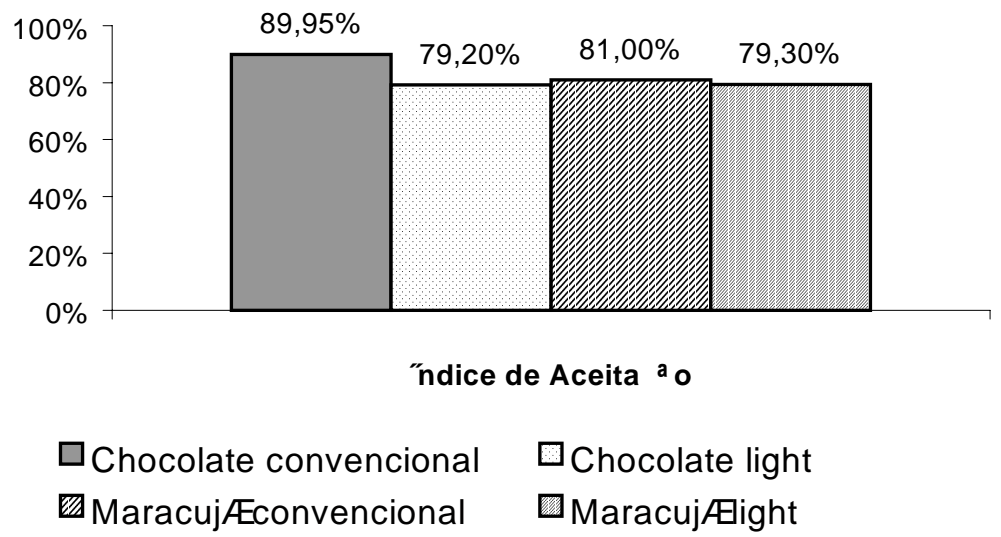

\subsection{RESULTADO DAS ANÁLISES FÍSICO-QUÍMICAS}

Os resultados das análises físico-químicas dos mousses de chocolate light e convencional são apresentados nas Figuras 10 e 11.

Foram obtidos valores calóricos de 154,10 e de $226,09 \mathrm{kcal} / \mathrm{kg}$ de produto para os mousses de chocolate light e convencional, respectivamente.

Verificou-se pequena diferença entre os teores de gordura dos mousses light e convencional. A redução calórica foi possibilitada pelo maior teor de umidade do produto light devido à adição de água para dissolução do espessante e do leite desnatado, que apresenta menor teor de sólidos que o creme de leite. 


\section{FIGURA 10 - COMPOSIÇÃO CENTESIMAL DO MOUSSE DE CHOCOLATE CONVENCIONAL}

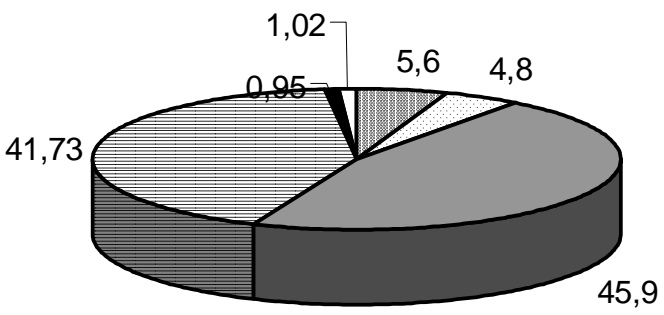

$\square$ Gordura

$\square$ Prote na

口Umidade

目Carboidratos

Fibras

$\square$ Cinzas

\section{FIGURA 11 - COMPOSIÇÃO CENTESIMAL DO MOUSSE DE CHOCOLATE LIGHT}

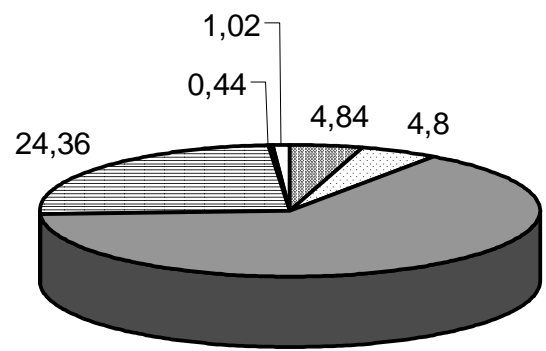

64,54
Gordura

$\square$ Prote na

$\square$ Umidade

目Carboidratos

Fibras

$\square$ Cinzas

DYMINSKI et al. (2000) estudaram as características físico-químicas de mousse de maracujá elaborado com substitutos de gorduras. Obtiveram redução média de 40,5\% no valor energético e de $93,6 \%$ no teor de gordura, comparativamente ao mousse padrão. Afirmaram que tais produtos podem ser classificados como light, tanto em relação ao valor energético como ao teor de gordura, sendo possível sua indicação como alimento adequado para pessoas preocupadas com a saúde e/ou obesas. 
A sucralose apresenta melhor desempenho que o aspartame quando aplicada em iogurtes, como maior resistência térmica, maior retenção da doçura, maior potencial de vida útil e maior consistência do produto. A adição de sucralose em qualquer etapa do processo não exige agitação ou operações de mistura indesejáveis que poderiam afetar a estabilidade e a textura do produto. Devido à estabilidade da sucralose também não é necessário incluir rótulos de advertência, nos produtos. A sucralose pode ser consumida por fenilcetonúricos sem inconvenientes, assim como por crianças e adolescentes, mulheres grávidas, pessoas idosas, diabéticos e pessoas com deficiência em sua defesa imunológica (CAMPOS, 2002).

\title{
4 CONCLUSÃO
}

Verificou-se preferência por sobremesa láctea nos sabores chocolate e maracujá, que fosse consistente e muito aerada, nas versões light ou convencional. As formulações que obtiveram maior índice de aceitação foram chocolate convencional $(89,99 \%)$ e light $(79,2 \%)$.

A aceitabilidade do mousse light sabor chocolate e a redução calórica de $31,8 \%$ em relação ao produto convencional permitiram concluir pela viabilidade da aplicação de sucralose nesse tipo de sobremesa.

\begin{abstract}

\section{SUCRALOSE IN THE DEVELOPMENT OF LIGHT MILKY DESSERTS}

The effect of the edulcorant sucralose in milky dessert type mousse was evaluated, by verifying the caloric reduction and the acceptability of the obtained product. A market research evidenced preference for milky dessert in chocolate or passion fruit flavors that were consistent, very aerated in light and conventional verisons. Four formulations were developed, in the two favorite flavors in the market research (chocolate and passion fruit), in the versions conventional and light. The formulations that obtained larger acceptance index were conventional chocolate and chocolate light. As results were obtained indexes of acceptance of $89,9 \%$ and $79,2 \%$ and caloric tenors of 226,09 $\mathrm{kcal} / \mathrm{kg}$ and $154,10 \mathrm{kcal} / \mathrm{kg}$ respectively for the mousse of conventional chocolate and light. A caloric reduction of $31,8 \%$ was verified, making viable the elaboration of dessert type mousse light using sucralose.
\end{abstract}

KEY-WORDS: MOUSSE; SUCRALOSE; MILKY DESSERT.

\section{REFERÊNCIAS}

1 AOAC. Association of Official Analytical Chemists. Official methods of analysis of the AOAC. $14^{\text {th }}$ ed. Washington, 1987. 
2 BRASIL. Ministério da Saúde. Secretaria de Vigilância Sanitária. Portaria no 27, de 13 de janeiro de 1998. Aprova Regulamento Técnico referente à Informação Nutricional Complementar. Diário Oficial [da] República Federativa do Brasil, Brasília, 15 jan. 1998, seção 1, p. 29514.

3 CAMPOS, M. B. Aplicação de sucralose em iogurtes e preparados de frutas para iogurtes. Food Ingredients, n. 19, p. 68-71, 2002.

4 CAMPOS, M. B. Sucralose: a revolução em adoçantes. Food Ingredients, n. 5, p.18-21, 2000.

5 CÂNDIDO, L. M. B.; CAMPOS, A. M. Alimentos para fins especiais: dietéticos. São Paulo: Varela, 1996. 432 p.

6 DYMINSKI, D. S.; WASZCYNSKYJ, N.; RIBANI, R. H.; MASSON, M. L. Características físico-químicas de musse de maracujá (Passiflora) elaborado com substitutos de gorduras. B. CEPPA, v. 18, n. 2, p. 267274, 2000.

7 LOURENÇO FILHO, R. C. B. Controle estatístico de qualidade. 17.ed. São Paulo: LTC, 1984. p. 204-205.

8 OSBORNE, D. R.; VOOGT, P. Análisis de los nutrientes de los alimentos. Zaragoza: Acribia, 1978. $290 \mathrm{p}$.

9 TEIXEIRA, E.; MEINERT, E. M.; BARBITTA, P. A. Análise sensorial de alimentos. Florianópolis: Editora UFSC, 1987. 180p.

10 VARNAM, A. R,; SUTHERLAND, J. P. Leche y productos lácteos: tecnología, química y microbiología. Zaragoza: Acribia, 1995. 476 p.

11 STATSOFT. Statistica 5.0. Tuse, 1999. 